\title{
La intimidad del aula frente a las lógicas de mercado
}

THE PRIVACY OF THE CLASSROOM AGAINST THE MARKET LOGIC

A INTIMIDADE DA SALA DE AULA DIANTE DAS LÓGICAS DO MERCADO

Liliana Rocío Torres López* / lilirotorresl@yahoo.com

\section{Resumen}

El presente documento reflexiona sobre los enlaces que se establecen entre las políticas internacionales y las nacionales y la forma como estos determinan las prácticas del aula en las instituciones educativas de Bogotá. A través de esta reflexión se visibiliza cómo se ha logrado implicar, devastadoramente, a la educación en las lógicas de mercado, por medio del discurso sobre la calidad y la productividad, lo cual ha afectado, especialmente, las formas de organización curricular y las prácticas cotidianas de los maestros de la Educación Media en la ciudad.

\section{Summary}

This paper reflects on the links established between the international and domestic policies, and how they determine classroom practices in educational institutions in Bogota. Through this reflection is made visible how it has been possible to imply, devastatingly, the education in the logic of the market through the discourse on quality and productivity, which has especially affected the forms of curricular organization and everyday practices of teachers of High School in the city.

\section{Resumo}

Este artigo reflete sobre os laços estabelecidos entre as políticas nacionais e internacionais, e como eles determinam as práticas de sala de aula nas instituições de ensino em Bogotá. Através desta reflexão se faz visível como se tem conseguido implicar, devastadoramente, a educação na lógica do mercado através do discurso sobre a qualidade e a produtividade, o que tem afectado, especialmente, as formas de organização curricular e as práticas cotidianas dos professores do ensino secundário na cidade.

\section{Palabras clave}

Mano de obra, política laboral, productividad laboral, educación básica, pertinencia de la educación, calidad de la educación.

\section{Key words}

Manpower, labor policy, labor productivity, basic education, educational relevance, educational quality.

\section{Palavras chave}

Trabalho, políticas de trabalho, a produtividade do trabalho, a educação básica, a relevância da educação, da qualidade da educação.

* Universidad Pedagógica Nacional. Comunicadora Social. Candidata a Magíster en Educación. 


\section{Los tránsitos de la lógica de mercado}

\section{Existe una estrecha relación entre las posi-} bilidades de inclusión social, el desarrollo de las microeconomías de los hogares y sus posibilidades de acceso a los bienes; así, en un trabajo conjunto realizado por la Comisión Económica para América Latina y el Caribe y la Corporación Latinobarómetro, se ha determinado que las líneas categoriales a través de las cuales se da dicha inclusión están determinadas por cuatro elementos: a) la posibilidad de tener una profesión u oficio, b) el poder contar con ingresos propios, c) el tener una propiedad, y d) el poder acceder a la educación superior (esta última, íntimamente relacionada con la primera); a estas categorías se suman las posibilidades de saber un idioma extranjero y saber utilizar un computador.

En este panorama de categorías y lo que cada una de ellas significa, permite visualizar que "las percepciones de exclusión no solamente se vinculan con la precariedad material" sino que tienen una estrecha relación con las posibilidades intelectuales y de conocimiento de las personas (Comisión Económica para América Latina y el Caribe, 2008).

No obstante lo anterior y a pesar de que eliminar la pobreza sea el primero de los objetivos del milenio trazados por las Naciones Unidas, en el año 2007, en América Latina, la incidencia de la pobreza y la indigencia alcanzan el 34,1\% de la población, lo cual significó la interrupción de la tendencia a la baja que en este sentido se había iniciado en el año 2002 (Op. cit.)

$Y$ es que las determinaciones económicas mundiales y las lógicas globales de mercado, definidas por los intereses personales de unos pocos, han definido las transformaciones que el mundo ha tenido durante los últimos tiempos con relación a todos los aspectos que constituyen el desarrollo de una sociedad: la salud, la cultura, la política, entre otras y, por supuesto, la educación se han instaurado así, en la lógica de la competitividad que se impone desde la preocupación de unos pocos por seguir adquiriendo sobre el sacrificio de la mayoría.

La respuesta que se ha dado ante esta situación, tiene como punto de partida el hacer entender a los gobiernos y a la población que si se aumentan las ofertas laborales se estaría dando respuesta a las necesidades de los más pobres; se afirma, entonces, que es necesario adoptar políticas macroeconómicas que incentiven la inversión productiva, aumenten las oportunidades de empleo y promuevan un ambiente social y económico estable, propicio para el desarrollo sostenido.

De esta forma, desde las políticas se crea una serie de exigencias al sector educativo en términos de generar estrategias que permitan viabilizar la ruta que permita ofrecer una Educación Secundaria de calidad y que propenda por la formación de sujetos que den respuesta a la exigencia de mano de obra cada vez mejor capacitada y educada (Op. cit.)

Así, se puede determinar que una línea que demanda un trabajo prioritario, para el desarrollo de los países latinoamericanos, está en la formación secundaria ya que en esta etapa de la vida de los individuos se requiere de la organización de alternaivas que, en un futuro próximo, le permitirán salir de la pobreza o no caer en ella, y porque la realización de los estudios secundarios "supone un salto cualitativo en el capital humano de la sociedad que permite crecer sobre la base de una mayor competitividad" (Op. cit.). Concretamente, se hace urgente fortalecer los procesos de cobertura y calidad en este nivel de la educación, para asegurar el acceso de los jóvenes a empleos de mayor productividad y así permitirles tener unos ingresos que les permitan mantenerse por encima de la línea de pobreza.

Como se puede ver, uno de los conceptos manejados por los entes decisorios de nivel internacional para caer cómodamente en las políticas nacionales y regionales, que tan cerca están de las mayorías, es el de calidad. Este concepto es favorable a tales intenciones porque su significación está tácita en la cotidianidad de las gentes, porque su requerimiento ya está instalado en los imaginarios colectivos y esto impedirá encontrar oposición debido a que se constituye en un propósito loable desde cualquier aspecto: la idea de "calidad" es uno de los objetivos centrales de la vida de los individuos.

\section{Politicas educativas nacionales y regionales: pretenciones de alimento para el aula}

Todos los sectores que constituyen el desarrollo social de un pueblo se ven exigidos frente a este panorama, $y$ cada uno de los países determina las líneas de su política particular, que orientarán las tendencias para transformarlo; de esta forma, las intenciones determinadas a nivel internacional, se particularizan en un nivel de realidad nacional y local, cuya escala es más asequible. 
La cuestión inicial es acerca de la ambigüedad que subsiste en el hecho de que uno de los objetivos del milenio sea erradicar la pobreza, no porque no sea un buen propósito, sino porque esto implica la ascendencia del discurso económico por encima de los que atañen a los otros sectores; trabajar para que no haya pobreza en el mundo significa haber reconocido que una de las principales fuentes de problemas en el mundo está directamente relacionada con la inequitativa distribución de la riqueza material entre los pueblos.

Trabajar por el cambio en este sentido implica que todos los sectores que determinan el desarrollo de una sociedad, incluyendo a la educación, habrán de trabajar en función de la economía y que las líneas de política que se establezcan con respecto a cada uno, habrán de orientarse hacia la transformación de la realidad que ésta ha impuesto.

Lo anterior supone, entonces, aceptar una lógica discursiva y práctica en la que lo más importante es el sostenimiento de unas intenciones desarrollistas desde la economía y no desde el capital humano; es aceptar que lo fundamental en una sociedad es la productividad basada en dos propósitos: a) aumentar los productos sin aumentar los insumos (en perspectiva de las lógicas de mercado y del consumo) y b) estructurar las lógicas laborales en los términos jerárquicamente organizados de empresario, trabajador y consumidor.

Esta situación tiene implicaciones claras y precisas para el caso del sector de la educación en Colombia. La primera de ellas es la ambigüedad dentro de la cual se debate, ya que los objetivos que la orientan pasan por una etapa transitoria que consiste en que, por un lado, es fundamental formar el pensamiento crítico en los individuos y enriquecer sus posibilidades intelectuales y, por otro, le resulta imperante obedecer a las lógicas del mercado dentro de las cuales se deben insertar o incluir los individuos que se están formando.

Se ha impuesto así una especie de afán con base en el cual se instauran, de forma confusa y desorientada, las políticas educativas determinadas en el Plan Sectorial de Educación y en el Plan Decenal Nacional de Educación. En estos dos documentos que guían las líneas de desarrollo de la educación nacional, departamental y regional, se establecen como categorías fundamentales la cobertura, la calidad, la pertinencia y la eficiencia (más claramente determinadas en el primero de estos planes), acá, entonces, se hace evidente, una vez más, lo imperativo del concepto calidad que sirve como conector entre los logros a nivel mundial y las metas a nivel nacional.

Las políticas nacionales son una herramienta fundamental para que los intereses que se mueven en los discursos económicos contemporáneos se materialicen en las líneas de trabajo regional y local; es así como para el caso de Bogotá se determina el plan de desarrollo del sector educativo dentro del cual se da respuesta a los requerimientos internacionalmente establecidos. De esta forma, el objetivo general de dicho plan se define como "garantizar a los niños, niñas, adolescentes y jóvenes de Bogotá las condiciones adecuadas para disfrutar del derecho a una educación de calidad que les sirva para el mejoramiento de la calidad de vida ..." (Alcaldía Mayor de Bogotá, 2008, p. 65).

Una de las necesidades que justifican el hecho de que la educación en Bogotá ha de estar al servicio del desarrollo económico de la región y del país, y no del desarrollo intelectual de la comunidad, está determinada por el hecho de que existe una "escasa articulación de la Educación Media con la Educación Superior y el Trabajo" de forma que se requiere "Construir una visión renovada de la Educación Media, que responda al requerimiento contemporáneo de los jóvenes para definir su proyecto de vida, que brinde posibilidades de trabajo dignas y formadoras de su identidad personal, que haga frente a las exigencias del mercado laboral generando nuevas alternativas para participar en el sistema productivo ..." (Alcaldía Mayor de Bogotá, 2008, p. 47).

Y como proyección de esta necesidad, los programas y proyectos que en este plan se determinan, apuntan con mayor puntualidad a responder los requerimientos descritos en la primera parte del presente documento. Es el caso de aquel que enuncia las "transformaciones pedagógicas para la calidad de la educación" dentro del que se propone que "la calidad educativa constituye una condición para mejorar las oportunidades, para lograr la inserción plena en la vida social y productiva, a la vez que contribuye de manera indiscutible al desarrollo de la sociedad en su conjunto" (Alcaldía Mayor de Bogotá, 2008, p. 72).

De esta forma se retoman conceptos como el de calidad para denominar el deber ser, tanto de la educación, como de la vida de las personas (educación de calidad para una calidad de vida) las cuales se verán positiva- 
mente afectadas gracias al ingreso de los estudiantes a la educación media. Los gobiernos entienden, como está visto, que a través de ésta los sujetos serán mejor formados para hacer parte de las cadenas productivas, haciendo evidente, una vez más, que el pensar en educación es, fundamentalmente, pensar en la economía.

Dos proyectos del Plan Sectorial de Educación de Bogotá están inmersos en los intereses del mercado impuestos a nivel internacional: implementación de la Especialización de la Educación Media y la Articulación.

\section{Los desafíos impuestos a la escuela y las respuestas desde la práctica pedagógica}

La intimidad del aula, que en términos de investigación educativa se denomina práctica pedagógica, está regulada, entonces, por las directrices impuestas la mayoría de las veces, por la puesta en marcha de un mecanismo de "gestión" frente al cual se dispone el trabajo de varios actores: las secretarías de educación, las entidades acompañantes y los directivos docentes.

Así pues, se asume que se comparten con los docentes los propósitos de su labor y, con escasa participación y discusión, ellos terminan siendo el vehículo que los materializa; los maestros acogen la impresionante pululación y desarticulación de los proyectos que agobian las oficinas de los directivos y lo que ellos mismos llaman "el nivel central", cuestionando, la mayoría de las veces, cuáles son las intenciones que orientan el diligenciamiento de formatos, la realización de reuniones, la transformación de los planes de estudio y los nodos que articulan unas dinámicas con otras.

Esta situación invita, y casi que obliga, a dar un giro en el desequilibrado deber ser de la escuela, específicamente en la Educación Media, aquel aspecto que se preocupa por formar sujetos críticos debe empezar a orientarse hacia el hecho de encontrar las formas de lograr mirar con cuidado lo que se les impone.

Esto significa que, ante las lógicas del mercado, es fundamental que el docente sepa que más allá de que sus estudiantes aprendan las competencias que le permitan acceder a un trabajo, resulta fundamental que sepan también que se requiere mejorar las condiciones de los procesos productivos a los que ellos ingresarán, o que resulta importante transformar la tendencia local, nacional y global a la inestabilidad laboral, o que se requiere mejorar el nivel de remuneración o las po- sibilidades, cada vez más escasas, de tener acceso a los sistemas de seguridad social (Comisión Económica para América Latina y el Caribe, 2008).

Con el fin de propender por la transformación de estas situaciones las acciones se deben desarrollar en contravía: si la lógica de mercado se impone desde el nivel mundial y se materializa, junto con sus efectos perversos, en las particularidades de las instituciones educativas, más exactamente en la intimidad del aula, la resistencia y la generación de alternativas habrá de darse desde éstas hacia aquellas; esto implica que la responsabilidad del docente es imprescindible y que, para poder hacer viable una transformación real, éste deberá estudiar y reconocer la lógica dentro de la que está su propia labor y aquella que se espera que ejerzan sus estudiantes.

Asimismo, las entidades acompañantes, bien sean instituciones de educación superior o grupos de investigación, tienen el compromiso de trabajar como puentes entre los docentes y las instancias decisorias tanto a nivel de los colegios, como en las instituciones donde se diseñan las políticas educativas (secretarías, ministerios), con el fin de lograr que se tengan en cuenta en la esencia de sus políticas, no sólo lo que se impone desde el exterior, sino también, las necesidades reales de los contextos en los que habitan estudiantes y docentes.

A su vez, estas entidades tendrán que reflexionar sobre su propia razón de ser, su compromiso con la transformación de los mecanismos laborales, de una parte, y de sus propios currículos y concepciones educativas, de otra. En otras palabras, a través del diálogo con las instituciones acompañadas, deberán lograr un reconocimiento detallado de las condiciones actuales de los jóvenes que cursan la Educación Media y actuar, hacia dentro y fuera de sí mismas, consecuentemente con lo aprendido.

La participación propositiva, dinámica y real de los docentes tanto de las instituciones educativas, como de las entidades acompañantes, entendidas como formadoras de formadores en ejercicio, específicamente, y de las nuevas generaciones de forma más general, es la alternativa que permitirá rescatar y articular la autonomía institucional, la independencia regional y la soberanía nacional, en términos educativos. 


\section{Diálogo del conocimiento}

Alcaldía Mayor de Bogotá. (2008). Plan Sectorial de Educación 2008 - 2012 - Educación de calidad para una Bogotá positiva. Bogotá: Alcaldía Mayor de Bogotá.

Albiñala, A. (1999). Geopolítica del Caos. Madrid: Le Monde Diplomatique España.

Bartolomé Pina, M. (1986). La Investigación Cooperativa. Bogotá, Educar.

Bogoya, D. (editor). (2003). Trazas y miradas. Evaluación y competencias. Bogotá, Colombia: Universidad Nacional de Colombia.

Bustamante, G. (2000). Ensayos sobre eduación en Colombia. Bogotá: Sociedad Colombiana de Pedagogía.

Catoriadis, C. (2002). "La cuestión de la autonomía social e individual". En: F. G. (Compilador). Pánico en la globalización (pp. 157-169). Bogotá: Fundación para la Investigación y la Cultura.

Chajín Flórez, M. (2004). Elementos para la construcción de una pedagogía dialógica. Ensayos disciplinares. Barranquilla, Colombia: Universidad Autónoma del Caribe.

Comisión Económica para América Latina y el Caribe. (2008). Panorama social de América Latina. En CEPAL, Panorama social de América Latina. Naciones Unidas, Santiago de Chile.

Foucault, M. (1981). Un diálogo sobre el poder. Madrid: Alianza.

Foucault, M., \& Deleuze, G. (s.f.). "Los intelectuales y el poder". En: M. Foucault Microfísica del poder. Madrid: La Epiqueta.

Grundy, S. (1994). Producto o praxis del currículo. Madrid: Ediciones Morata.

Kemmis, S. (2009). "El currículo: más alla de la teoría de la reproducción". En: Morin, E. Para una política de la civilización. Madrid: Paidós.

Park, P. (1992). "¿QQué es la investigación acción participativa. Perspectivas teóricas y metodológicas". En: Salazar, M. C. (Comp). La Investigación-acción participativa. Inicios y Desarrollos. (pp.135- 174). Colombia: Editorial Popular. OEI, Quinto Centenario.

Polet, F. \& Houtart, F. (2001). El otro Davos - globalización de resistencias y de luchas - Madrid: Editorial Popular S.A.

Productivamente. (s.f.). Recuperado el 8 de Mayo de 2009, de http://www.productivamente.org/productividad/about.php

Quijano, A. (2000). "Colonialidad del poder y clasificación social". En: Journal of world - systems research.

Ramírez, L. A. (2004). Discurso y lenguaje en la educación y la pedagogía. Bogotá: Cooperativa Editorial Magisterio.

Universidad Nacional de Colombia. (2009). Criterios, objetivos y estrategias de educación media especializada en el Distrito Capital. Bogotá: Universidad Nacional de Colombia.

Virno, P. (2003). Gramática de la Multitud. Madrid: Traficantes de Sueños.
La intimidad del aula frente a las lógicas de mercado es un artículo escrito por la maestra Liliana Torres que esboza de forma crítica algunos aspectos problemáticas de la ecuación actual, tales como: la contradicción intrínseca que encierra la educación que se orienta desde la práctica pedagógica, lo que ella denomina intimidad en el aula, en relación con las lógicas internacionales, nacionales y locales de mercado que ordenan los contenidos, el enfoque y las estructuras que deben formarse, como lo son la calidad, la productividad y el progreso. Desde este punto de vista el artículo nos recuerda que la escuela, cualquiera que sea su nivel, obedece a la misión de ser una institución moderna, y que desde su génesis se empodera como una necesidad política desde la cual se pretende lograr el desarrollo y progreso de un país. La pregunta que subyace en toda la argumentación presente en el artículo, y que cuestiona la relación sistema económico, práctica pedagógica es, cómo formar sujetos críticos que cuestionen las lógicas del mercado en las que necesariamente tendrán que insertarse una vez como terminan sus estudios. Pareciera entonces que la sociedad avanza en un doble discurso, la superestructura económica que determina qué debe enseñarse, cómo, por qué, etc., $y$ un discurso humanista que cuestiona el progreso, la falsa noción de progreso, de desarrollo, la esclavitud laboral, la pobreza, los pros y contras del sistema de cambio, en fin, debemos empeñarnos, como lo señala la autora, en tener una participación activa en las discusiones políticas que desde nuestro orilla puedan darse, necesaria por cierto, no debemos olvidar que si las políticas internacionales exigen a la escuela formar personas competentes para el mercado o la empresa, nuestra respuesta es aún mayor, pues nosotros formamos la empresa más grande e importante que es la mente, que no formamos solo sujetos de saber sino sujetos políticos y de deseo, y que nuestro deseo como educadores será siempre ser críticos de los vejámenes de una sociedad cada vez más esclavista.

Edilson Silva Liévano 